\title{
Burnout in healthcare: a sign of death by overwork for doctors in China
}

The authors of this letter (BMJ 2019;366:15582, doi:10.1136/ bmj.15582) requested that the address of second author, Z-L Chen, be updated to Reproductive Medical Centre, Central
Hospital of Wuhan, Tongji Medical College, Huazhong University of Science and Technology, Wuhan, China. This has been updated online. 